\title{
Comparative analysis on digital surface model of urban area from Sentinel-1 SAR interferometry and aerial photogrammetry for disaster mitigation plan
}

\author{
Fathoni Usman ${ }^{1,2 *}$, Nanda $^{2}$, Rita Nasmirayanti ${ }^{2}$, and Josapath Tetuko Sri Sumantyo ${ }^{3}$ \\ ${ }^{1}$ Institute of Energy Infrastructure, The Uniten University, 43000 Kajang, Malaysia \\ ${ }^{2}$ Department of Civil Engineering, Faculty of Engineering, Putra Indonesia University - YPTK, 25145, Padang, Indonesia \\ ${ }^{3}$ Centre for Environmental Remote Sensing, Chiba University, Chiba, Japan
}

\begin{abstract}
This paper presents an effort to evaluate the generated digital elevation model (DEM) from an active sensor onboard satellite of Sentinel-1A and from aerial photos taken using an unmanned aerial vehicle (UAV). The objective is to compare the quality of generated DEM and review the processes for disaster mitigation and prevention plans application. The radar data acquisition used in this study is pair of SLCtype radar data. The interferogram is processed from the coherence and the phase of complex data of the pair radar imageries. Meanwhile, aerial photography was taken within the smaller urban area in Padang City. The photogrammetry process to generate the DEM was conducted using the structure from motion (SfM) technique. The quality and procedures are reviewed by comparing the DEM products with other publicly available DEM data from DEMNAS, SRTM, and AW3D. This study found that generating the DEM from Sentinel-1 interferometry SAR is a challenging process. The product is unmatched and has lower quality compared to available DEM data due to several identified factors. In contrast, high computational cost photogrammetry produced good quality DEM if sufficient ground control points (GCP) were set.
\end{abstract}

\section{Introduction}

The satellite era on remote sensing has given the availability of Digital Elevation Model (DEM) from its active and passive sensor on board. The application extends beyond the earthwork analysis and design for civil engineering works to various spatial analysis and environmental sciences $[1,2]$. It has been used in hydrology, geology, and geomorphology research to model and predicts land deformation for disaster mitigation and many others. The accuracy and extend of uses depend on the topographic information's quality spatial resolution and temporal resolution. The detail spatial resolution ranged from $10 \mathrm{~cm}, 5-15 \mathrm{~m}, 30-90$ $\mathrm{m}$, and $1 \mathrm{~km}$ [3]. Different data types were used to generate the DEM from land surveying works, high spots from aerial photos stereography, 3D laser scanning (LIDAR), to satellite remote sensing data.

Meanwhile, data acquisition of aerial imageries has been replacing traditional land surveying products to a certain extent $[4,5]$. The metropolitans and urban areas have static features on the earth's surface that predominantly shape the surface model compared to bare land. Contributions from static objects covering the landscape affect the generated DEMs. As a result, elevation refers to the height of the surface rather than the elevation of the terrain [6].

\subsection{SAR Interferometry}

Synthetic Aperture Radar Interferometry (InSAR) is an advanced technique to retrieve past and continuously earth surface deformation in a millimeter observation scale [7]. This technique has been applied in landslide monitoring and prediction, land subsidence due to natural and human activities in high temporal resolution [8]. However, on rapid movement and changing of surface, limited by the temporal baseline, the interpretation of the surface deformation hampered the performance [7]. The challenge has made the use of the InSAR method in the derivation of DEM less published [2].

\subsection{Structure from Motion}

Aerial photogrammetry has been used in complementary and, to a certain extent, replacing conventional land surveying in generating 3D surface modeling [9]. The Unmanned Aerial Vehicle (UAV), commonly called a drone, provides indispensable assistance, fast and precise, and relatively cost-saving [5]. The drone technology becomes more user-friendly to navigate and can access highly inaccessible terrain and landscape. The drone is becoming more affordable due to collaborative efforts to use the photogrammetry technology on aerial imagery and

\footnotetext{
*Corresponding author: fathoni@uniten.edu.my
} 
its quick advancement and significant demand from retail customers.

Generating DEM using photogrammetry starts with flying a drone at a specific elevation and takes a high degree of overlapping multiple aerial photos. The flying path should cover the intended area by sticking the images to reconstruct the $3 \mathrm{D}$ of the surface object. The coordinates of objects are calculated using triangulation of coordinates from two coordinate systems, i.e., the camera coordinate and the world coordinate [10].

\section{Method and Materials}

This study evaluates the generated DEM from the interferometry technique of SAR and aerial photogrammetry. The remote sensing data of SAR cover the Padang City area. The drone that took the aerial photo has flown where the site was identified as responsive to earthquakes. Pada City is the capital of West Sumatra Province. From the two fault zones of Sumatra Fault Zone (SFZ) and Mentawai Fault Zone (MFZ), the distances are about $80 \mathrm{~km}$ and $120 \mathrm{~km}$, respectively, to the urban area of Padang City. Fig. 1 shows the fault zones and subduction trance along Sumatra, with the circle shape representing the earthquake's location and its magnitude $[11,12]$.

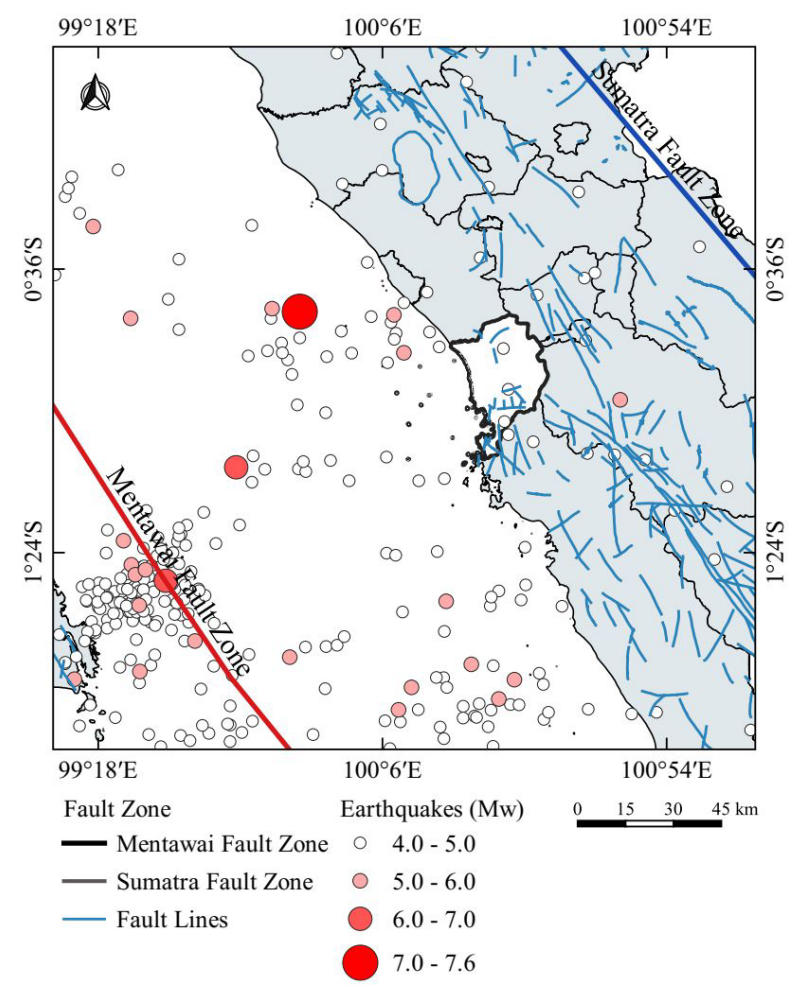

Fig. 1. Fault zones of Sumatra and Mentawai with the earthquake events that occur from August 2009 to June 2021.

The radar data are collected from the Copernicus program website that is available publicly. The data are C band Sentinel-1A mission, single look complex (SLC) type with acquisition swath mode of interferometric width (IW) in descending pass VV co-polarization (transmitting and receiving the beam in vertical polarization). Sentinel1 was designed to measure the surface changing using interferometry. The pairs of imagery will have narrow orbital tubes [2]. The pairs of radar data in different perpendicular baselines (Bperp) and temporal baseline (Btemp) for this study are listed in Table 1. Those radar data will be used to study the changes in the coherence over the time of its temporal baseline.

Table 1. The information of Bperp and Btemp on pairs of SAR data in this study.

\begin{tabular}{|c|c|c|c|c|}
\hline $\begin{array}{c}\text { Acquisition } \\
\text { Date }\end{array}$ & Role & $\begin{array}{c}\text { Perpendicular } \\
\text { Baseline } \\
\text { (m) }\end{array}$ & $\begin{array}{c}\text { Temporal } \\
\text { Baseline } \\
\text { (days) } \\
\end{array}$ & $\begin{array}{c}\text { Delta } \\
\text { fDC } \\
(\mathbf{H z}) \\
\end{array}$ \\
\hline $15 / 09 / 2019$ & Master & \multicolumn{3}{|c|}{-} \\
\hline $20 / 03 / 2021$ & Slave & -25.35 & -552 & -1.60 \\
\hline $15 / 09 / 2019$ & Master & \\
\hline $16 / 08 / 2020$ & Slave & 36.30 & -336 & -0.23 \\
\hline $15 / 10 / 2020$ & Master & \multicolumn{3}{|c|}{ - } \\
\hline $20 / 03 / 2021$ & Slave & -76.97 & -156 & -0.22 \\
\hline $15 / 10 / 2020$ & Master & \multicolumn{3}{|c|}{ 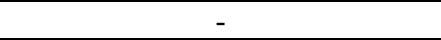 } \\
\hline $02 / 12 / 2020$ & Slave & -19.86 & -48 & -0.11 \\
\hline $23 / 07 / 2020$ & Master & \\
\hline $16 / 08 / 2020$ & Slave & -26.83 & -24 & -0.04 \\
\hline $08 / 03 / 2021$ & Master & \multicolumn{3}{|c|}{-} \\
\hline $20 / 03 / 2021$ & Slave & -28.41 & -12 & -2.77 \\
\hline
\end{tabular}

Processing radar SLC radar data starts with selecting a pair of radar imagery to set as master and slave and reduces it into a single sub-swath with the chosen number of bursts. The orbit file is then applied and followed by backscattered geocoding. The interferogram is created using the InSAR technique, which multiplies the amplitudes and calculates the phase difference. Back geocoding and Enhanced Spectral Diversity (ESD) is used to improve the image quality. A debursted interferogram is the result of this procedure by applying the TOP SAR debursted command. The process is continued by setting the multi-look parameters and improving the image in Goldstein phase filtering. A wrapped interferogram is the finished product for the phase unwrapping process [13, 14]. The process in SeNtinel Application Platform (SNAP) 8.0 is shown in Fig. 2. The steps process the reflected wave, the backscattered from the radar sensor that emitted microwave radiation. The backscattered signal contains information on phase and amplitude. In [13] and [15], comprehensive technical tutorials for processing the SLC data from Sentinel 1 mission were explained using InSAR to generate DEM in SNAP.

Meanwhile, the basic SfM approach begins with reconstructing a sparse point cloud using feature-based matching to produce a tie point cloud. As a result of this step, multi-stereo pairs are constructed using epipolar geometry, with the collected images aligned and their relative orientation established. Outlier detection is used iteratively to remove incorrect point matches. The camera's interior and exterior orientation parameters are determined simultaneously by minimizing a global reprojection error [16]. After each tie point's space resection and intersection are resolved, a sparse point cloud with $3 \mathrm{D}$ coordinates can be generated in any coordinate system. Second, surveying or determining the locations and orientations of camera exposure stations are used as common georeferencing methods. A least-squares 
adjustment of the 3D coordinates of the sparse point cloud is made using this data along with the tie points.

Creating dense point clouds from scratch is stage three of the process. The disparities at all pixels are estimated using the previously determined epipolar geometry of the photogrammetric block from the first phase in the third stage, which uses image matching techniques. A 3D surface free of unanticipated anomalies is created using gradient-based and energy-minimization techniques, which back-project pixels onto all images and triangulate them. The SfM pipeline creates a 3D surface's raw representation as RGB-coloured dense point clouds. The process of generating DEM in Agisoft Photoscan software is presented in Fig. 3.

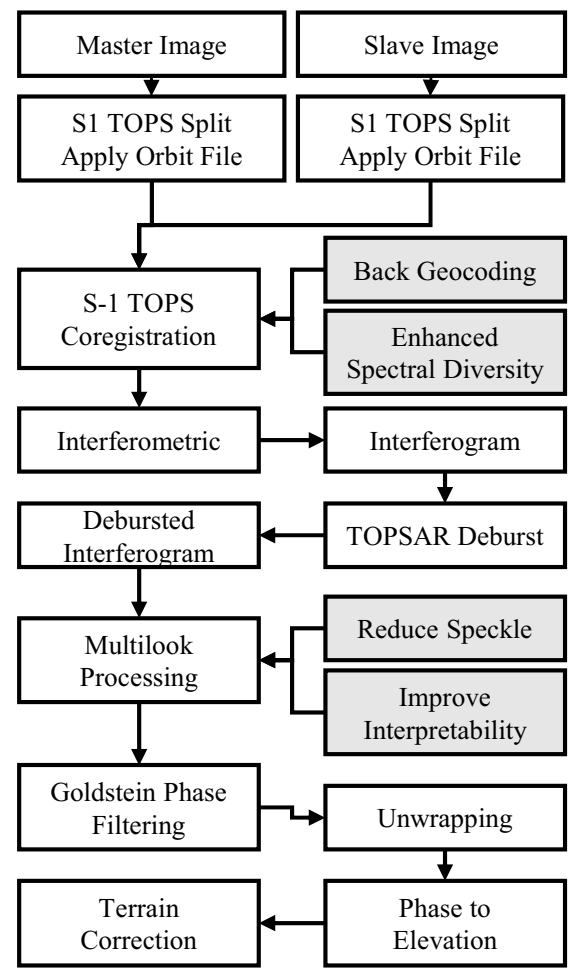

Fig. 2. The DEM generation processes in SNAP 8.0

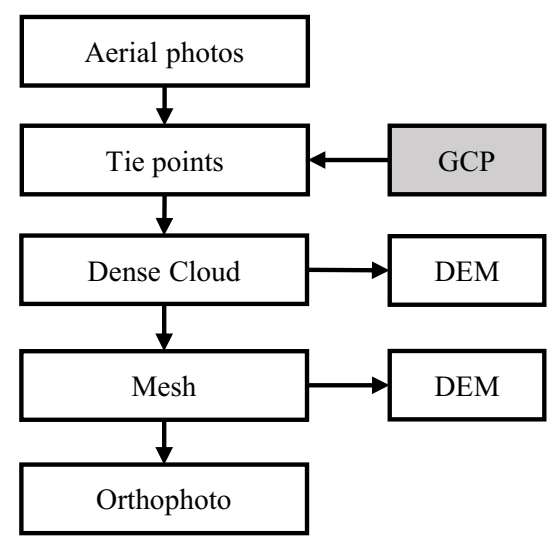

Fig. 3. The DEM generation processes in Agisoft Photoscan Professional 1.4.5

Equation 1 is used to calculate the ground sampling distance, where $\bar{D}$ is the ground sampling distance, $S_{w}$ is the sensor width of the camera, $H$ is the flight height, $F_{r}$ is the focal length of the camera, and $I_{w}$ is the image width of the camera sensor [10]. The precisions obtained from SfM with close-range photogrammetry principles for convergent imagery estimate the achievable coordinate accuracy, $\overline{\sigma_{c}}$ using Equation 2 for individual targets viewed in $k$ images where $q$ is a factor representing the photogrammetric network geometry, $\bar{\sigma}_{c}$ is the precision of image measurements, and $d$ is the principle of convergent imagery measured the same as the lens's focal length [4].

$$
\begin{gathered}
\bar{D}=S_{w} \times H \times I_{w} \times \frac{100}{F_{r}} \\
\bar{\sigma}_{c}=\frac{q \bar{D}}{\sqrt{k} d} \bar{\sigma}_{l}
\end{gathered}
$$

Three pairs of SLC radar data used for DEM generation in the urban area of Padang City are listed in Table 2. The perpendicular baseline of more than $100 \mathrm{~m}$ is selected, and the closest temporal baseline is 48 days. The radar data selection assisted using the Baseline search command in ASF data search. Finding a near temporal baseline with a perpendicular baseline of more than 150 $\mathrm{m}$ will be difficult if manually conducted in SNAP using the InSAR stack overview command for the radar data.

Table 2. The pairs of SAR data for DEM generation in this study.

\begin{tabular}{|c|c|c|c|}
\hline $\begin{array}{c}\text { Acquisition } \\
\text { Date }\end{array}$ & Role & $\begin{array}{c}\text { Perpendicular } \\
\text { Baseline } \\
\text { (m) }\end{array}$ & $\begin{array}{c}\text { Temporal } \\
\text { Baseline } \\
\text { (days) }\end{array}$ \\
\hline $11 / 08 / 2021$ & Master & \multicolumn{2}{|c|}{} \\
\hline $28 / 09 / 2021$ & Slave & 153 & 48 \\
\hline $12 / 06 / 2021$ & Master & \multicolumn{2}{|c|}{} \\
\hline $11 / 08 / 2021$ & Slave & 109 & 60 \\
\hline $15 / 02 / 2021$ & Master & \multicolumn{2}{|l}{} \\
\hline $12 / 04 / 2021$ & Slave & 147 & 48 \\
\hline
\end{tabular}

In preparing the data for SfM, a drone was set to fly about mid-day to minimize the shadow. There are 1329 images were taken with a flying altitude of $31.9 \mathrm{~m}$. Nine ground control points (GCP) were placed (in with circle) within the coverage area of $0.126 \mathrm{~km}^{2}$ as shown in Fig. 4. Fig. 4 shows the number of images overlapping, with most interior regions having more than five overlapped images.
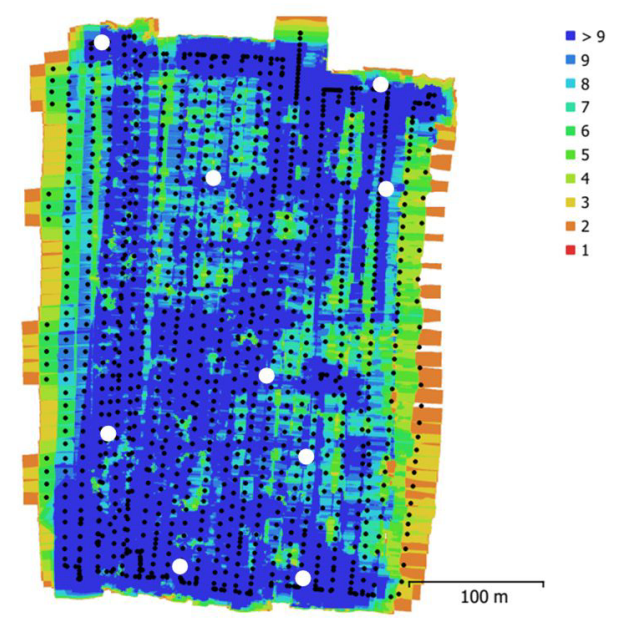

Fig. 4. The GCPs on the overlapped images within the flight coverage area. 


\section{Discussion}

This paper presents a development process of a DEM using publicly available Sentinel-1 data from the Copernicus program of the European Satellite Agency. The procedures are discussed in section 2 of this paper. The radar data of SLC that carry radiometric information have been selected with different Bperp and Btemp. The primary purpose is to evaluate the effect on the quality of coherence in developing a high-quality InSAR. It is seen that as the temporal baseline increase, the decorrelation happens to reduce the coherence. Coherence is a valuable indicator of an interferogram's quality. Fig. 5 shows the subset of coherence images on the district of Kota Padang. The box plot graph in Fig. 6 shows the reduction in coherence value on areas denser with buildings and more vegetated regions of the northern part of Kota Padang Regency.
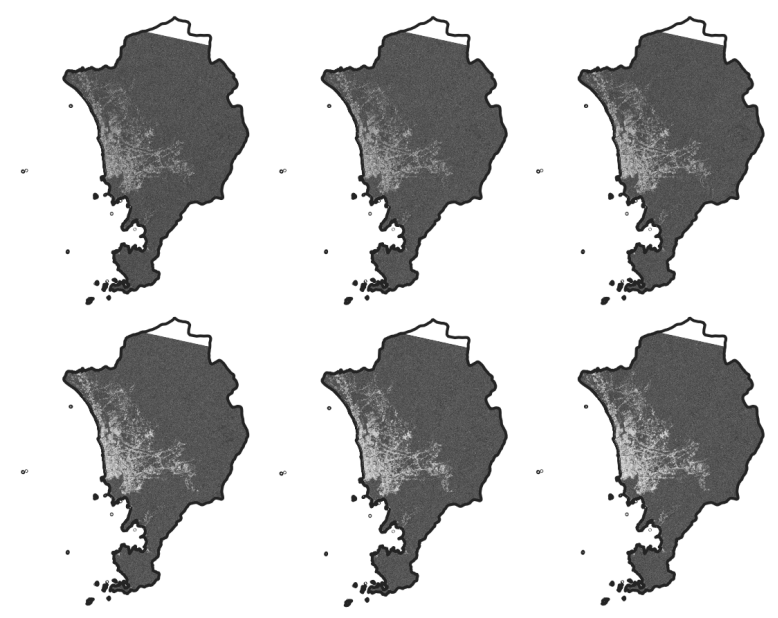

Fig. 5. The subset of coherence data on the district of Kota Padang Regency.

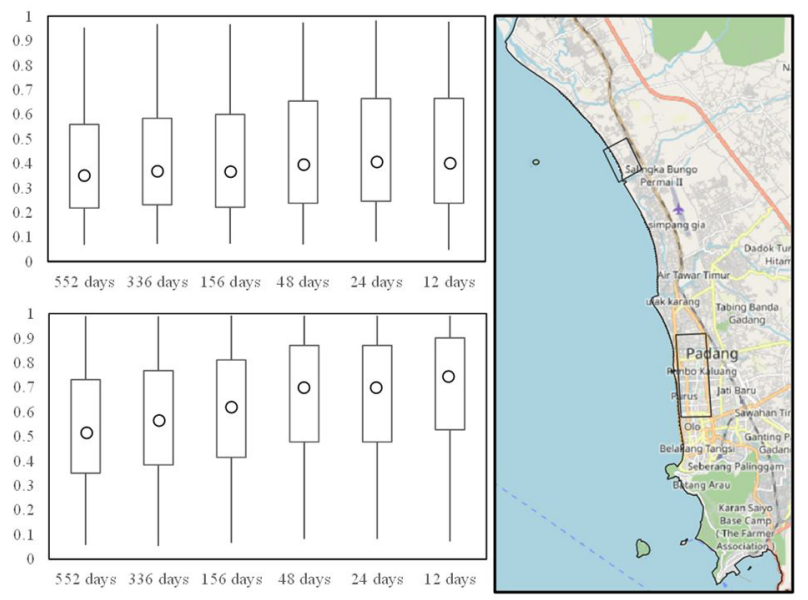

Fig. 6. Box plot of coherence value over the temporal baseline on the different areas with less vegetation on the top and denser vegetation on the bottom.

Consideration should be given to short temporal baselines, acceptable perpendicular baselines, and appropriate atmospheric conditions for data acquisition as well. Because moisture in the atmosphere creates phase delays and degrades measurement quality, the radar data suggested being collected on dry and least rainy days [17]. Between May and July, the Padang City area experienced the least rainfall. The Baseline search function on the ASF data search website did not find radar data within these three months with a short temporal baseline and a perpendicular baseline of more than $150 \mathrm{~m}$. Adjustment for atmospheric delays was not used in this study but reducing the adaptive filter exponent to 0.3 in Goldstein's filtering avoided the cross-like pattern in phase on the urban area [13].

In general, the root-mean-square error (RMSE) values are incredibly high at $391.37 \mathrm{~m}$ in the urban area of Padang City, which is densely vegetated in its northern area and scattered vegetation in its southern urban area. Fig. 7 shows the elevation difference of the DEM generated from Sentinel-1 radar data taken on April, August, and September 2021 compared to DEM from DEMNAS, AW3D, and SRTM. The DEM of DEMNAS has a 0.27 -arcsecond resolution, which is $8 \mathrm{~m}$ spatial resolution, and Both AW3D and SRTM have $30 \mathrm{~m}$ spatial resolution. Even though using the DEM generation procedures of InSAR in SNAP, the spatial resolution of Sentinel-1's DEM is $13 \mathrm{~m}$, the C band wave has its limitation. The $\mathrm{C}$ band Sentinel-1 has the challenge to penetrate dense vegetation and irregular shape of building formations. The hillshade view of DEMs from this study is shown in Fig. 8.

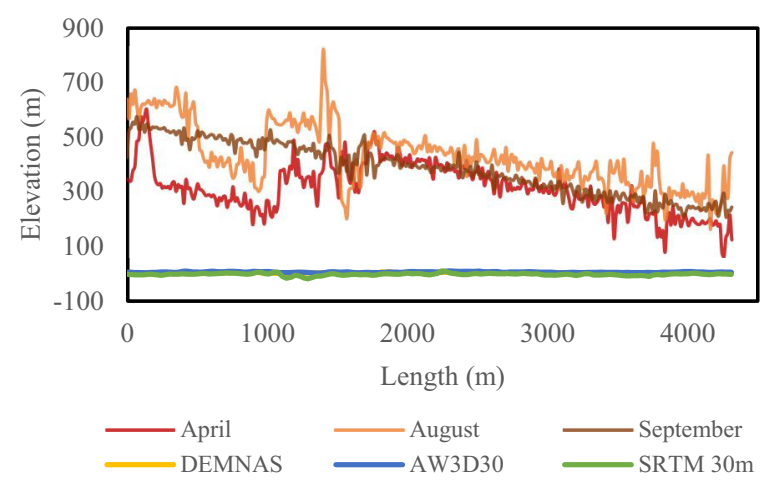

Fig. 7. The elevation difference of the DEM from Sentinel-1 from three acquisition dates.
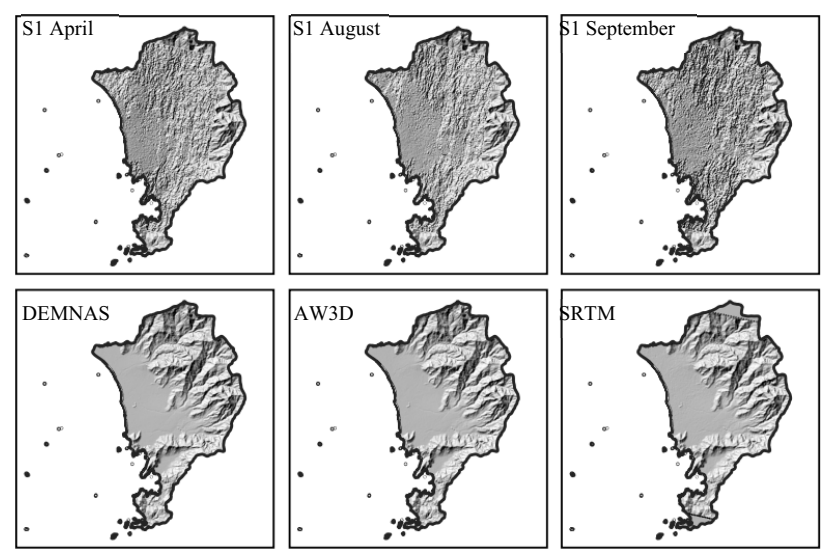

Fig. 8. The elevation difference of the DEM from Sentinel-1 (S1) from three acquisition dates in hillshade view. 
On the other hand, the SfM application in generating point clouds to produce the DEM has been conducted to compare its products with and without ground control points (GCP). In this study, the GPCs tap to the DEM from DEMNAS, and the generated DEMs were taken from four products of point cloud and mesh with cleaning and without cleaning processes. Fig. 9 shows the difference between DEM products. The DEM generated without GCP and relied on the onboard positioning, and orientation only has an RMSE of $1.96 \mathrm{~m}$ on line A, 2.06 $\mathrm{m}$ on line $\mathrm{B}$, and $3.56 \mathrm{~m}$ on line $\mathrm{C}$. The placement of GCP near the perimeter of the coverage area will avoid the curvature error on the DEM products as at line B in Fig. 9. The number of GCP to quality of DEM products has a strong correlation, and it was suggested to add 2 GCPs for every hectare of land [18, 19]. Additionally, the placement of GCP close to the perimeter of the coverage area is recommended based on this study finding when a consumer-based UAV was used to take aerial images.
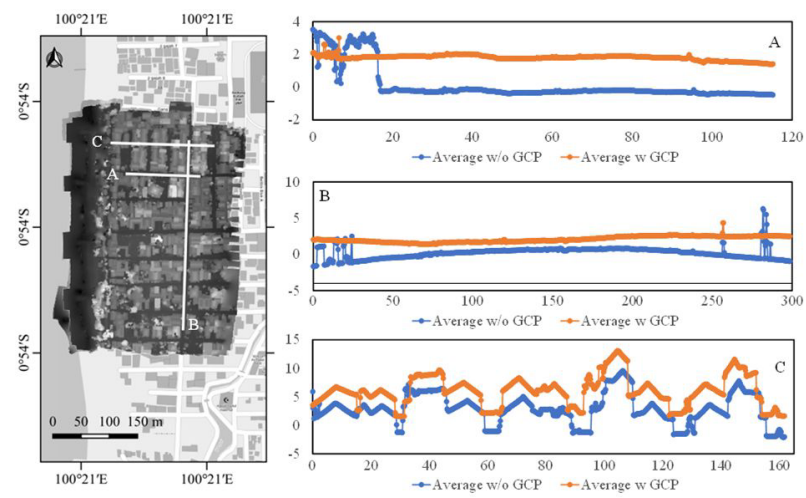

Fig. 9. The average elevation comparison between DEM products with GCPs and without GCPs.

From these two approaches of DEM derivation, the need to have updated DEM for various applications, especially for disaster mitigation plans, is still an utmost effort for urban areas where changes are still dominant due to new development and urbanization. The satellite remote sensing has extensive area coverage. While aerial photography using drones have become more affordable, they made an opportunity to provide updated DEM available. Unfortunately, derivation of DEM from Sentinel-1 radar data with its $\mathrm{C}$ band and available suitable baseline has limited the use. This study has identified and added some recommendations in generating DEM using both approaches.

\section{Conclusion}

Due to numerous usage and application, generating DEM is one of the essential information in geospatial analysis. Generating DEM from radar data is a challenging effort. This study presents an effort to generate DEM for specific purposes of a disaster mitigation plan. The Available Sentinel-1 SLC data for interferometry SAR analysis has many advantages. The Spatio-temporal of 6 days for a tandem mission and 12 days for a single mission provide a good spatial resolution. The generated DEMs were compared with the publicly available DEMs of DEMNAS, SRTM, and AW3D. The Sentinel-1 is designed for Differential SAR Interferometry to extract phase into displacement in a millimeter detail order. In generating the DEM for an urban area with vegetation canopy scattered in between buildings, it was found that lower coherence derives a low quality of generated DEM. The Sentinel-1 cannot produce a good quality DEM because of the low perpendicular baseline (Bperp) value of pair imageries. The Bperp suggested being in between 150 to $300 \mathrm{~m}$ which is difficult to get. The other reason for the low quality of generated DEM is Sentinel-1 uses C-band that cannot penetrate denser vegetation canopy in tropical areas. The atmospheric artifact also becomes an issue. Advance processes of developing the DEM from Sentinel-1 SLC data in SNAP Desktop software is a steep learning curve to explore.

The authors would like to thank the Centre for Environmental Remote Sensing (CEReS), Chiba University, Japan, for the CEReS Overseas Joint Research Program 2021 (CI21-111) to Universitas Putra Indonesia - YPTK Padang, Indonesia.

\section{References}

1. H. Tsunetaka, N. Hotta, Y. S. Hayakawa, and F. Imaizumi, Prog. Earth Planet. Sci. 7, 1 (2020)

2. A. Braun, Open Geosci. 13, 1 (2021)

3. J. Jasiewicz and T. F. Stepinski, Geomorphology, 182 (2013)

4. M. R. James and S. Robson, J. Geophys. Res. Earth Surf. 117, 3 (2012)

5. P. Sestras, Ștefan Bilașco, S. Roșca, B. Dudic, A. Hysa, and V. Spalević, Remote Sens. 13, 3, (2021)

6. I. Borlaf-Mena, M. Santoro, L. Villard, O. Badea, and M. A. Tanase, Remote Sens. 12, 18 (2020)

7. Q. Meng, F. Raspini, P. Confuorto, Y. Peng, and H. Liu, EGU Gen. Assem. (2021)

8. S. Amariena Hamim, F. Usman, and A. Kurnia Shalihat, Adv. Eng. Res. 187 (2019)

9. J. A. Navarro, N. Algeet, A. Fernández-Landa, J. Esteban, P. Rodríguez-Noriega, and M. L. GuillénCliment, Remote Sens. 11, 1 (2019)

10. P. Razi et al., J. Phys. Conf. Ser. 1876, 1 (2021)

11. M. Diament, Geology 20, 3 (1992)

12. R. Hall, M. A. Cottam, and M. E. J. Wilson, The SE Asian Gateway: History and Tectonics of the Australia-Asia Collision. (Bath: The Geological Society Publishing House, 2011)

13. A. Braun, Sentinel-1 Toolbox DEM generation with Sentinel-1 Workflow and challenges, (Skywathc, 2020).

14. F. Usman, A. Syamsir, and J. Melasari, Mapping of Earthquake-Induced Land Deformation on Urban Area Using Interferometric Synthetic Aperture Radar Data of Sentinel-1, in Recent Advances in Earthquake Engineering, Lecture Notes in Civil Engineering 175 (2022) 
15. ASF DAAC, How to Create a DEM using Sentinel-1 Data (ASF DAAC, 2018)

16. E. F. Berra and M. V. Peppa, Advances and challenges of UAV SFM MVS photogrammetry and remote sensing: Short review, 2020 IEEE Lat. Am. GRSS ISPRS Remote Sens. Conf. LAGIRS 2020 Proc. (2020)
17. A. Mohammadi et al., Sensors (Switzerland), 20, 24 (2020)

18. S. Coveney and K. Roberts, Int. J. Remote Sens. 38, 8-10 (2017)

19. L. Hashemi-Beni, J. Jones, G. Thompson, C. Johnson, and A. Gebrehiwot, Sensors (Switzerland) 18, $11(2018)$ 